Musées, Patrimoine et Culture scientifiques et techniques

$161 \mid 2015$

septembre-octobre 2015

\title{
Faire vivre la gratuité
}

\section{Claude Fourteau}

URL : http://journals.openedition.org/ocim/1573

DOI : $10.4000 /$ ocim. 1573

ISSN : 2108-646X

\section{Éditeur}

OCIM

Édition imprimée

Date de publication : 1 septembre 2015

Pagination : $30-33$

ISSN : 0994-1908

Référence électronique

Claude Fourteau, «Faire vivre la gratuité », La Lettre de I'OCIM [En ligne], 161 | 2015, mis en ligne le 01 septembre 2016, consulté le 01 mai 2019. URL : http://journals.openedition.org/ocim/1573 ; DOI : 10.4000/ocim. 1573

Ce document a été généré automatiquement le 1 mai 2019.

Tous droits réservés 


\section{Faire vivre la gratuité}

\section{Claude Fourteau}

L'article (disponible sur www.ocim.fr, rubrique La Lettre de l'OCIM) montrait notamment que la gratuité nécessite un accompagnement de médiation auprès des publics.

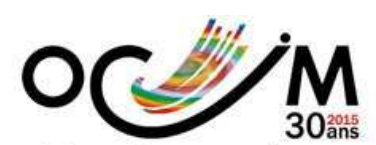

La gratuité, point aveugle des politiques culturelles

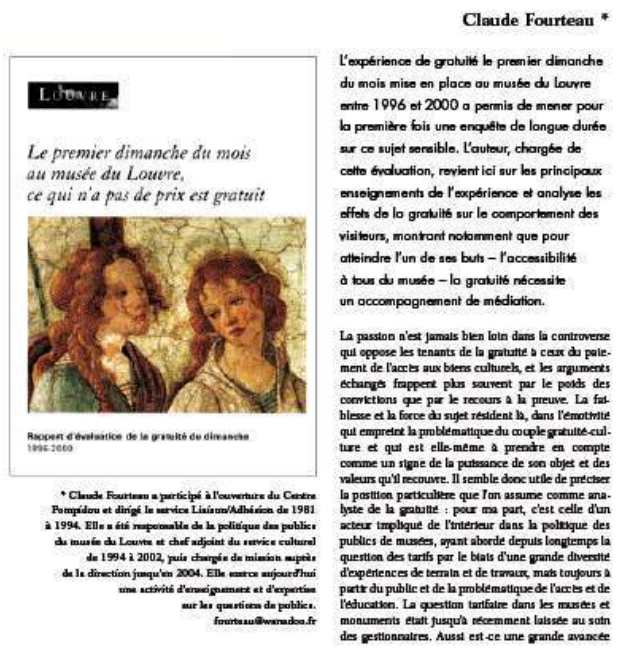

wamen atrean............15

1 Il y a 20 ans, le retour expérimental de la gratuité pour tous le premier dimanche du mois au Louvre a pris à rebours la longue évolution qui, de 1921 jusqu'en 1995 avait peu à peu fini par abroger en France ce grand principe de gratuité des musées. Entre la visée d'éducation du peuple et le rationalisme économique, s'est insérée la "modernisation de l'action publique $»^{1}$ qui pousse les musées à développer leurs ressources propres. Il est donc particulièrement intéressant de suivre, dans ce contexte, ce qu'est devenue la 
renaissance de la gratuité : a-t-elle résisté au temps, conquis les publics, confirmé ou infirmé son utilité et sa valeur?

\section{Une propagation irrésistible}

2 Ce qui s'impose d'abord, au cours de ces deux décennies, est le développement continu et diversifié de la gratuité. L'expérimentation au Louvre s'est prolongée de 1995 à 2000, cinq années au cours desquelles un socle d'évaluation nettement positif s'est constitué sur les effets de la gratuité, relevés par enquêtes continues; et sur ces bases, en 2000, le ministère de la Culture élargit la gratuité du dimanche à tous les musées nationaux et aux monuments historiques. Par effet d'entraînement, municipalités et collectivités territoriales, dans leur majorité, l'adoptent à leur tour - ou vont au-delà - tel Paris qui rend totalement gratuits, en 2001, ses musées municipaux, suivi par une douzaine de villes en régions. Enfin, le record d'affluence des jeunes les jours de gratuité confirme, devant leur ardeur, la nécessité de lever le frein financier : en 2009, l'exonération totale d'accès aux collections est allouée aux Européens de moins de 26 ans. On peut ainsi considérer qu'aujourd'hui la gratuité, sous différentes formes, est acquise et mobilisée dans l'essentiel du réseau public des musées et monuments en France : une reconquête pleine d'avenir !

3 À l'étranger aussi les expériences se sont multipliées, allant souvent bien au-delà du cas français. Dès 2001 la surprise est venue du Royaume-Uni, où le Labour a décrété la gratuité totale de l'accès aux collections des musées nationaux - exemple rapidement suivi par la Suède. On connaissait la singularité des musées de la Smithsonian Institution à Washington, depuis toujours gratuits, sur le modèle initial anglais, et qui le restent. Et il est intéressant de constater que parmi les 15 musées les plus fréquentés du monde ${ }^{2}$, neuf sont gratuits - dont huit appartiennent à ces deux pays, ce qui confirme bien l'effet de la gratuité sur le nombre de visiteurs. Plus considérable encore, par son effet potentiel sur une population immense et largement ignorante de la pratique des musées, est la décision de la Chine, depuis 2011, de rendre gratuits pour tous ses bibliothèques et ses musées publics, « initiant ainsi la réforme des services de l'État dans le domaine des arts et de la culture $»^{3}$, en partie sur un modèle européen.

\section{L'actualité du débat}

4 En parallèle de son intégration dans la politique des publics, la gratuité est redevenue sujet de débats, d'études, de parutions, de l'analyse quantitative à la conviction engagée, de l'étude des représentations à la stratégie tarifaire ou à l'expérience de terrain. Les enquêtes s'accordent toutes pour reconnaitre l'accroissement considérable de fréquentation que provoque la gratuité, mais les interprétations divergent sur les preuves de son efficacité sur la composition du public.

5 L'enquête de MORI, institut de sondage qui s'est attaché à mesurer les effets de la gratuité complète instaurée au Royaume-Uni depuis 2001, a corroboré de près les résultats relevés pendant cinq ans par le Louvre : l'accroissement considérable de la fréquentation $(+60 \%)$, mais surtout l'amplification de l'ancrage national $(+230 \%)$, qui a englobé toutes les catégories sociales et fait apparaître surtout l'élargissement spectaculaire des visiteurs jeunes, mais aussi des familles, des primo-visiteurs, des couches sociales peu familières de 
la pratique du musée. L'étude menée en 2008 sur l'expérimentation de la gratuité totale de 14 musées et monuments répartis en France atteste de «l'effet accélérateur de la gratuité sur la démocratisation »; $47 \%$ des visiteurs ne seraient pas venus sans la gratuité et $83 \%$ des visiteurs interrogés la plébiscitent.

6 En revanche, bien des arguments négatifs font poids dans le débat et mettent la gratuité en position de défensive : le «baromètre des publics du Louvre ", avec le temps, voit les effets positifs s'inverser et c'est dans ce contexte que le musée vient de supprimer le dimanche gratuit durant "la haute période touristique », (un jour de gratuité par mois est ramené à six mois par an), rejoignant ainsi le mode de gratuité saisonnier appliqué par Versailles et les monuments historiques.

7 Les critiques se portent sur les arguments suivants : les effets d'aubaine, la persistance des inégalités, la supériorité en nombre des touristes étrangers, l'excès d'affluence... Peutêtre n'est-il pas inutile de revisiter ces problématiques en d'autres termes.

\section{Le dimanche gratuit : quel effet d'aubaine?}

8 L'argument dénonce comme "effet d'aubaine » la présence nombreuse de visiteurs qui seraient déjà des familiers de musées et monuments, et qui pourraient payer. C'est ainsi que la journée de gratuité serait détournée de son but par des «profiteurs». Cet argument économique, souvent utilisé par Madame Thatcher pour supprimer la gratuité, n'est pas neuf, mais il ne correspond en rien à la définition de la démocratisation dans la pensée française. Pour quelle raison un habitué ne pourrait-il pas user lui aussi de la gratuité pour tous? Faut-il oser penser que le dimanche gratuit ne serait fait que pour les pauvres de la culture, dans un élan de charité ? Ne voit-on pas, à l'œil nu et sans même le besoin d'enquête, que la diversité des âges et la mixité sociale sont bien présentes et même remarquables au cours de cette journée qui libère la visite ? Rappelons la définition donnée par Malraux aux missions de son ministère: «Rendre accessibles les œuvres capitales de l'humanité ... au plus grand nombre possible de Français ». La démocratisation, il faut l'entendre d'abord comme le rapprochement du plus grand nombre, le public rassemblé. La gratuité du dimanche, quand elle a été restaurée a été définie comme « un signe fort qui fait comprendre que la culture s'adresse à tous $»^{4}$. Et ce signe reçoit en réponse une mobilisation hors de l'ordinaire, une abondance de visiteurs divers qui ont en commun un sentiment d'appartenance. La gratuité pour tous, ainsi posée, est sans conteste une très puissante expérience de lien social à partir d'un ordre symbolique, ainsi que nous en avons précédemment tenté l'analyse. Suffit-elle à modifier subitement les pratiques? Bien sûr que non, mais elle ouvre des horizons et des chances : elle n'exclut pas !

\section{Qu'en est-il des inégalités ?}

9 Un second argument est à considérer pour sa base méthodologique. Il concerne la persistance des inégalités entre catégories sociales, que la gratuité ne compenserait pas. La répartition par pourcentage des différentes catégories socioprofessionnelles de visiteurs du dimanche gratuit, serait à peu près comparable à celle du dimanche payant, marquée donc par la prédominance des catégories supérieures. Or ce n'est pas le cas. L'expérimentation de la gratuité de 14 musées et monuments en 2008, qui a l'avantage de 
refléter petites et grandes institutions dans le cadre national, confirme les indicateurs socioculturels d'une démocratisation : $28 \%$ de la population enquêtée est constituée de primo-visiteurs; les catégories populaires sont les plus mobilisées (avec les jeunes) et comptent pour 1/3. Leur bond en nombre est d'autant plus remarquable que c'est justement la moitié de primo-visiteurs qui viennent. Quelle autre mesure que la gratuité entraine-t-elle un tel effet? On a vu que l'accroissement de tous les visiteurs est considérable mais les pourcentages écrasent les nombres et la définition de la démocratisation, que donne l'analyse sociologique, veut que si tous les milieux sociaux progressent à allure égale, il n'y a pas de progrès démocratique, car pas de « rattrapage ». On en conclut l'échec de la gratuité. N'est-ce pas la méthode qui appelle le résultat ${ }^{5}$ ?

\section{La mondialisation des visiteurs}

10 Le musée est avec le monument l'institution culturelle qui reçoit le public le plus cosmopolite et les visiteurs étrangers constituent bien souvent les $2 / 3$ de la fréquentation des plus grands établissements. Historiquement, c'est le tourisme de masse qui a provoqué la mise en cause de la gratuité et finalement son abrogation. Cependant, autant une politique culturelle s'est organisée pour les ressortissants nationaux, autant persiste un vide sidéral à l'égard des étrangers. Les vagues touristiques ont toujours été dépréciées et considérées surtout pour leur apport de revenus. Des efforts importants ont été faits pour améliorer l'accueil, le confort, l'organisation des visites, les documents en langues étrangères, un contrôle sur la qualité des accompagnateurs, mais de responsabilité culturelle point. "Assurer le rayonnement de la France » ne semble être conçu que comme une politique culturelle hors sol, alors que l'essentiel de ce rayonnement se constitue sur notre territoire, auprès des millions de visiteurs qui le foulent. Le jour de gratuité devrait être un de ces moments où le visiteur étranger se sente intégré à partager un héritage universel. De la gratuité pour tous, lui aussi se souviendra.

11 L'autre volet qui explique la supériorité en nombre des étrangers vient de la carence de communication; tandis que les tours operators informent les touristes de la gratuité, les institutions et l'État ont cessé de promouvoir avec l'engagement nécessaire le message que «ce qui n'a pas de prix est gratuit». Il ne faut pas s'étonner que les étrangers en viennent à surpasser de nouveau en nombre les résidents nationaux, en ce jour particulier. Aujourd'hui, $40 \%$ de la population ignore l'existence de la gratuité.

\section{Les nuisances de l'affluence}

Gérer des foules immenses : là encore la difficulté n'est pas nouvelle. Au milieu du XIX siècle, le Louvre gratuit recevait jusqu'à 20000 visiteurs le dimanche; considérablement agrandi, il en reçoit près du double aujourd'hui... Sans doute bien des moyens ont dû être tentés pour mieux répartir les visiteurs par des propositions de circuits alternatifs et séduisants. Mais simplement, pourquoi ne pas décider d'augmenter les horaires d'ouverture ? Maintenir la fermeture de l'entrée des collections à $17 \mathrm{~h} 30$, avec seulement deux nocturnes par semaine semble bien inadapté à la vie moderne et à l'affluence générale du musée. 
Cela dit, la grande affluence touche essentiellement les très grands établissements. Et comment ne pas voir que la plupart des musées et monuments sont en recherche de publics et que cette journée est pour eux une corne d'abondance, renouvelée chaque mois. Quelle chance que de rencontrer tant de visiteurs pour arpenter les collections, si souvent délaissées au profit des expositions! Quant aux risques courus par les œuvres, il ne semble pas que le peuple du dimanche ait jamais rien détérioré. Bien plus, la foule a des vertus de protection pour le visiteur néophyte. Pour s'apprivoiser il suffit de se fondre. Le visiteur nouveau apprend de la simple présence du visiteur expérimenté, il adapte son comportement, baisse la voix, écoute par bribes un conférencier de passage, regarde avec surprise travailler le copiste, le maçon, l'architecte... C'est ainsi que se familiarise, à l'abri de la foule, un visiteur en devenir.

\section{Quelles perspectives?}

La gratuité a retrouvé une place qui est sans doute irréversible, malgré les critiques qui subsistent. Elle fait partie de l'esprit du temps, qui est au partage et à l'ouverture. Quelle forme va-t-elle revêtir? Permanente, exceptionnelle, catégorielle? Elle a la vertu de l'évidence pour les jeunes, qui considèrent les institutions culturelles comme des lieux d'éducation gratuits. Mais ils constituent le cas d'exception, car ils ont soif de connaissance, d'art, de culture, sans en avoir les moyens. Pour eux la gratuité ciblée a toute sa place. Impossible d'obtenir la gratuité pour tous, alertent les économistes qui ne voient pas comment pourrait se remplacer le manque à gagner de la billetterie. Conserver gratuit le premier dimanche du mois parait une solution médiane. La force du dimanche est d'être un signe compris par tous, avec une gratuité attractive car exceptionnelle, et éducative car régulière. Mesure modeste dira-ton? Non, car elle propose douze visites par an qui peuvent se transformer en projet régulier d'avenir pour des apprentisvisiteurs.

15 La gratuité n'est pas faite pour gagner de l'argent, on peut s'en douter, mais pour gagner des visiteurs : des visiteurs occasionnels pour qu'ils viennent plus souvent, des publics que ni la famille ni l'école n'ont familiarisés à la visite de musées ou de monuments et qui découvrent qu'ils n'en sont pas exclus, des jeunes curieux de tout, des foules rassemblées sans objectifs consuméristes... Pour que s'accomplisse ce projet au niveau qui doit l'être, il faut que les responsables des institutions, les politiques, les analystes le portent dans le cadre de ce que les anglo-saxons appellent joliment « the raison d'être » du musée.

\section{BIBLIOGRAPHIE}

Anderson, M. Less is more, Museum news, vol. 93, $\mathrm{n}^{\circ}$ 2, 2014.

Benhamou, F. Généraliser la gratuité des musées nationaux ? Esprit, nº 6, 2008.

Ceroux, B. et Eidelman, J. La gratuité dans les musées et monuments en France : quelques indicateurs de mobilisation des visiteurs, Culture études, 2009-2. 
Fourteau, C. La gratuité au Bois dormant... Cinq ans de gratuité du dimanche au Louvre 1996-2000. Paris : Musée du Louvre, 2001.

Fourteau, C. La gratuité, point aveugle des politiques culturelles, La Lettre de l'OCIM, nº 111, 2007.

Gombault, A., Bourgeon-Renault, D. et al. La gratuité des musées et des monuments côté publics Représentations, projets d'usage et comportement des publics. Paris : La Documentation française, 2006.

Octobre, S. Politique des publics et politique tarifaire, Musées \& collections publiques de France, $\mathrm{n}$ - 248, 2006.

Robatel, N. et Krebs, A. Démocratisation culturelle : l'intervention publique en débat. Paris : La Documentation française, 2008.

Tobelem, J.-M. La culture mise à prix. Paris : L'Harmattan, 2005.

\section{NOTES}

1. Le Comité interministériel pour la modernisation de l'action publique (CIMAP) 18 décembre 2013.

2. Most popular Museums and galleries, 2013, Source AECOM (repris par Economist.com/graphic detail).

3. People's Daily, 18 février 2011, Yu Qun, Chef du Département social de la Culture, Pékin.

4. Philippe Douste Blazy, ministre de la Culture, présentant le projet de la gratuité du premier dimanche de chaque mois au Conseil de Direction du musée du Louvre, décembre 1995.

5. À titre d'exemple, les chiffres suivants, tirés du dossier d'évaluation de la gratuité au Louvre 1996-2000, font apparaître divers angles de mesure qui portent à la réflexion et à la diversité des interprétations : parmi les visiteurs d'île-de-France, sont primo-visiteurs un dimanche ordinaire : $12 \%$ des cadres supérieurs (CS), $24 \%$ des professions intermédiaires - hors enseignants - (PI), 36 $\%$ des employés/ouvriers (E0). Sur 100 primo-visiteurs, la différence en pourcentage entre dimanche ordinaire ou gratuit est faible : 18/18 (CS), 29/25 (PI), 53/57 (EO). En nombre de primovisiteurs, entre dimanche payant et gratuit, on compte 145/270 (CS), 240/370 (PI), 425/850 (EO).

\section{RÉSUMÉS}

À l'occasion des 30 ans de l'OCIM, La Lettre de l'OCIM propose à ses lecteurs, dans chaque numéro de l'année 2015, un retour sur un article marquant de l'histoire de la revue. À partir d'un corpus d'articles choisis par la rédaction de La Lettre de l'OCIM, les membres du comité des Publications de l'OCIM ont sélectionné plusieurs contributions.

Dans cette perspective, il a été demandé à l'auteur ou à un expert du domaine de revisiter la problématique exposée dans l'article à la lueur des changements intervenus - notamment dans les pratiques professionnelles - depuis son écriture et de proposer des éléments prospectifs sur la question.

Claude Fourteau revient sur son article "La gratuité, point aveugle des politiques culturelles" publié en mai-juin 2007 dans le $n^{\circ} 111$ de La Lettre de l'OCIM et dans lequel elle tirait les premiers 
enseignements de l'expérience de gratuité menée au musée du Louvre en analysant les effets de cette gratuité sur le comportement des visiteurs.

INDEX

Mots-clés : Gratuité, politique culturelle, public

\section{AUTEUR}

\section{CLAUDE FOURTEAU}

responsable de la politique des publics du musée du Louvre et chef adjoint du service culturel de 1994 à 2002, puis chargée de mission auprès de la direction jusqu'en 2004

fourteau@wanadoo.fr 